\title{
Adesão ao tratamento da dor crônica e o locus de controle da saúde*
}

\author{
COMPLIANCE WITH THE TREATMENT OF CHRONIC PAIN AND HEALTH CONTROL LOCUS \\ ADHESIÓN AL TRATAMIENTO DEL COLOR CRÓNICO Y EL LOCUS DE CONTROL DE LA SALUD
}

\author{
Geana Paula Kurita1 ${ }^{1}$ Cibele Andrucioli de Mattos Pimenta²
}

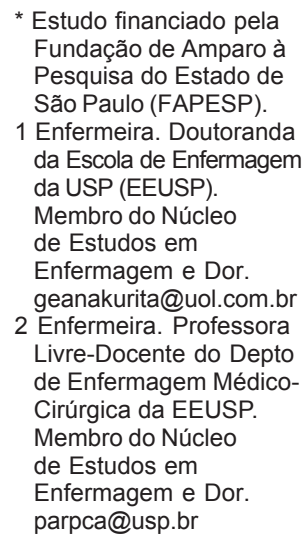

* Estudo financiado pela Fundação de Amparo à Pesquisa do Estado de São Paulo (FAPESP).

1 Enfermeira. Doutoranda da Escola de Enfermagem da USP (EEUSP).

Membro do Núcleo de Estudos em

Enfermagem e Dor. geanakurita@uol.com.br

2 Enfermeira. Professora Livre-Docente do Depto de Enfermagem MédicoCirúrgica da EEUSP. Membro do Núcleo de Estudos em Enfermagem e Dor. parpca@usp.br

\author{
RESUMO \\ Objetivou-se avaliar a adesão \\ dos doentes com dor crônica \\ não oncológica ao tratamento \\ e identificar relações entre a \\ crença de controle da saúde e \\ a adesão. Trinta doentes \\ foram avaliados durante seis \\ meses, em cinco entrevistas. \\ A adesão foi classificada em \\ plena, parcial e não adesão, \\ e foi calculado o Índice de \\ Acerto de Ingestão Medica- \\ mentosa (IAIM). A crença de \\ controle da saúde foi avaliada \\ por meio da Escala de Locus \\ de Controle da Saúde. Obser- \\ varam-se altos índices de \\ adesão parcial e não adesão \\ ao tratamento $(40,0 \%$ a $56,7 \%)$, \\ médias do IAIM entre $57,2 \%$ e \\ $69,5 \%$, e que doentes com \\ crenças de maior "interna- \\ lidade" aderiram menos ao \\ tratamento.
}

\begin{abstract}
The objective was to assess compliance of patients with non-oncologic pain and identify relationships between beliefs of health control and compliance. Thirty patients were evaluated over a period of six months, in five interviews. Compliance was classified as total, partial and non-compliance, and the Index of Correct Medication Ingestion (ICMI) was calculated. The belief about health control was evaluated by the Multidimensional Health Locus of Control Scale. High rate of partial compliance and noncompliance with the treatment (40.0\% to $56.7 \%$ ), ICMI means between $57.2 \%$ and $69.5 \%$ were observed, and that patients with higher "internality" beliefs were less compliant with the treatment.
\end{abstract}

\section{KEYWORDS}

Pain.

Patient compliance.

Internal-external control.

\section{RESUMEN}

El estudio tuvo como objetivo analizar la adhesión de enfermos con dolor crónico no oncológico al tratamiento e identificar relaciones entre la creencia de control de la salud y la adhesión. Fueron evaluados treinta enfermos durante seis meses, en cinco entrevistas. La adhesión fue clasificada en plena, parcialy no adhesión, y fue calculado el Índice de Acierto de Ingestión Medicamentosa (IAIM). La creencia de control de la salud fue evaluada por medio de la Escala de Locus de control de la salud. Se observaron altos indices de adhesión parcialy no adhesión al tratamiento (40.0\% a $56.7 \%)$, promedios del IAIM entre $57.2 \%$ y $69.5 \%$, y que enfermos con creencias de mayor internalidad se adhirieron menos al tratamiento.

\section{PALABRAS CLAVE}

Dolor.

Cooperación del paciente. Control interno-externo. 


\section{INTRODUÇÃO}

A não adesão ao tratamento é um dos maiores problemas em saúde pública ${ }^{(1)}$. Aspectos socioculturais estão relacionados a ela e, embora relevantes, são pouco explorados. O locus de controle da saúde e as crenças frente à dor são aspectos importantes no manejo da dor crônica.

O locus de controle é um modelo que propõe ser a crença do indivíduo (motivação interna e externa) determina a ação a ser tomada. Quem acredita que os resultados ao menos em parte, são dependentes das ações tomadas, é considerado internamente orientado. Aquele que tem orientação externa geralmente não acredita ou crê pouco na relação entre resultado e ação individual ${ }^{(2)}$.

O modelo cognitivo comportamental considera que os valores, as atitudes, as crenças, entre outros fatores, determinam a experiência de dor ${ }^{(3)}$. As crenças influenciam o doente na percepção e expressão da dor, em como lidar com ela e no manejo do tratamento.

A compreensão dos aspectos socioculturais que norteiam a adesão pode auxiliar na definição do que se recomendar ao doente, na comunicação entre o paciente e o profissional e em maior seguimento à terapia proposta. Este estudo teve o objetivo de avaliar a adesão dos doentes ao tratamento da dor crônica não oncológica e identificar relações entre a crença de controle da saúde e a adesão.

\section{CASUÍSTICA E MÉTODO}

Trata-se de um estudo prospectivo longitudinal com 30 doentes com dor crônica não oncológica em tratamento no Ambulatório de Dor da Clínica Neurológica do HC-FMUSP, ao longo de seis meses, entre abril/1999 e maio/2000. Os critérios de inclusão adotados foram: ter iniciado tratamento entre o período citado anteriormente, ter idade igual ou superior a 18 anos, apresentar queixa de dor há pelo menos seis meses, possuir condições físicas e independência para locomoção, demonstrar capacidade de compreensão e verbalização adequadas para participar do estudo e residir na cidade de São Paulo. O estudo foi aprovado pela Comissão de Ética para Análise de Projetos de Pesquisa do Hospital das Clínicas da Faculdade de Medicina da Universidade de São Paulo e todos os doentes assinaram termo de consentimento. A idade dos doentes variou entre 24 e 76 anos (média $=52,6$ anos, mediana $=52$ e dp=14,44) e $86,7 \%$ foram do sexo feminino.

A avaliação da adesão foi feita mediante o relato do doente e classificada em plena (uso correto de todas as medicações), parcial (uso de pelo menos uma das medicações) e não adesão (não utilização de nenhuma das medicações prescritas). Também foi calculado o Índice de Acerto de Ingestão Medicamentosa (IAIM) que consiste no percentual de medicação corretamente ingerida considerando-se o total de medicação prescrita. A intensidade da dor foi avaliada por meio da escala numérica de 0 a 10 , onde 0 significa ausência de dor e 10 a pior dor imaginável ${ }^{(4)} \mathrm{e}$ foi tratada como uma variável descritiva, de caracterização da amostra.

Foram feitas cinco avaliações para identificar o comportamento de adesão e a intensidade da dor, e três para avaliar o locus de controle da saúde. A primeira avaliação ocorreu entre 7 e 15 dias após o início do tratamento. As demais avaliações foram estabelecidas contabilizando-se o número de dias após essa primeira avaliação isto é, a segunda, entre 15 e 25 dias, a terceira, entre 45 e 55 dias, a quarta, entre 105 e 115 dias, e a quinta avaliação, entre 165 e 175 dias. O Locus de Controle da Saúde foi avaliado na primeira, terceira e quinta avaliações, e a intensidade da dor em todas.

O Locus de Controle da Saúde (LCS) compreende a percepção das pessoas sobre a fonte de controle dos acontecimentos ${ }^{(2)} \mathrm{e}$ foi avaliado por meio da Escala de Locus de Controle da Saúde (ELCS) $)^{(2,5)}$. Esse questionário permite a identificação de três dimensões do locus de controle: a dimensão pessoal (convicção do sujeito de controlar a sua vida - subescala "internalidade"), a dimensão social (outros sujeitos poderosos como fonte de controle - subescala "outros poderosos") e a dimensão impessoal (sorte, acaso e destino como responsáveis pela situação - subescala "acaso"). A partir dessas dimensões pode-se identificar dois extremos de controle: externo ("outros poderosos", o "acaso", a sorte e o destino) e interno (o próprio sujeito ${ }^{(6)}$. Na avaliação da escala de Locus de Controle da Saúde, os escores
Adesão ao tratamento da dor crônica e o locus de contrôle da saúde 
Geana Paula Kurita Cibele A. de Mattos Pimenta variam entre 0 e 30 , sendo que quanto maior o valor do escore mais o indivíduo acredita em uma das fontes de poder. $\mathrm{O}$ índice de "internalidade total" é calculado por meio de uma fórmula específica que engloba as subescalas envolvidas na "internalidade"(7).

$$
\mathrm{IT}=\mathrm{I}-\frac{\mathrm{OP}+\mathrm{A}}{2}
$$

IT=intenalidade total $\mathrm{I}=$ escore de internalidade $\mathrm{OP}=$ escore de outros poderosos $\mathrm{A}=$ escore de acaso

Foram realizados testes estatísticos paramétricos e não paramétricos para analisar a existência de mudanças, ao longo do tempo, nas variáveis: nível de adesão (ANOVA e Friedman); locus de controle (ANOVA e Friedman) e intensidade da dor (ANOVA e Friedman). Os testes Correlação, de Pearson e de Spearman, foram utilizados para analisar as relações entre adesão e locus de controle. O nível de significância foi determinado em $5 \%$.

\section{RESULTADOS}

Na avaliação da adesão de 30 doentes, ao longo de seis meses, observou-se que o número de plenamente aderentes variou entre $43,3 \%$ a $56,7 \%$, nos cinco momentos. O número de plenamente aderentes foi prevalente em quatro das cinco avaliações. No entanto, quando somados, os parcialmente aderentes e os não aderentes (variação entre $40,0 \%$ a $56,7 \%$ ) foram superiores aos plenamente aderentes em quatro das cinco avaliações. Ao final do período de seis meses, 27 doentes foram parcialmente aderentes e apenas 3 plenamente aderentes.

Tabela 1 - Distribuição dos doentes com dor crônica de acordo com a adesão ao tratamento medicamentoso, ao longo de seis meses (São Paulo, 2000)

\begin{tabular}{|c|c|c|c|c|c|c|c|c|c|c|c|c|}
\hline \multirow[t]{3}{*}{ Adesão } & \multicolumn{10}{|c|}{ Momentos de avaliação } & \multirow{2}{*}{\multicolumn{2}{|c|}{$\begin{array}{c}\text { Ao final } \\
\text { de } 6 \text { meses }\end{array}$}} \\
\hline & \multicolumn{2}{|c|}{1} & \multicolumn{2}{|c|}{2} & \multicolumn{2}{|c|}{3} & \multicolumn{2}{|c|}{4} & \multicolumn{2}{|c|}{5} & & \\
\hline & $\mathrm{n}$ & $\%$ & $\mathrm{n}$ & $\%$ & $\mathrm{n}$ & $\%$ & $\mathrm{n}$ & $\%$ & $\mathrm{n}$ & $\%$ & $\mathrm{n}$ & $\%$ \\
\hline Plena & 13 & 43,3 & 11 & 36,7 & 14 & 46,7 & 17 & 56,7 & 14 & 46,7 & 3 & 10,0 \\
\hline Parcial & 12 & 40,0 & 14 & 46,7 & 11 & 36,7 & 7 & 23,3 & 10 & 33,3 & 27 & 90,0 \\
\hline Não & 5 & 16,7 & 5 & 16,7 & 4 & 13,3 & 5 & 16,7 & 6 & 20,0 & - & - \\
\hline Total & 30 & 100,0 & 30 & 100,0 & $29^{*}$ & 96,7 & $29^{*}$ & 96,7 & 30 & 100,0 & 30 & 100,0 \\
\hline
\end{tabular}

* Um doente faltou à entrevista

O maior Índice de Acerto de Ingestão Medicamentosa (IAIM) foi de $69,5 \%$ na quarta avaliação e o menor foi de $57,2 \%$ na primeira avaliação (Tabela 2). Os testes estatísticos aplicados revelaram que não houve diferença no IAIM entre as avaliações (Tabela 2), isto é, a adesão, expressa pelo IAIM, não variou ao longo de seis meses.

Tabela 2 - Estatística descritiva e analítica do Índice de Acerto de Ingestão Medicamentosa (IAIM) ao longo deseis meses (São Paulo, 2000)

\begin{tabular}{|c|c|c|c|c|c|c|c|c|}
\hline \multirow{2}{*}{$\begin{array}{l}\text { IAIM } \\
\%\end{array}$} & \multicolumn{6}{|c|}{ Momentos de avaliação } & \multicolumn{2}{|c|}{ Significância P } \\
\hline & 1 & 2 & 3 & 4 & 5 & $\begin{array}{c}\text { Ao final } \\
\text { de } 6 \text { meses }\end{array}$ & ANOVA & Friedman \\
\hline Média & 57,2 & 58,3 & 60,3 & 69,5 & 65,6 & 62,5 & - & - \\
\hline Mediana & 66,7 & 66,7 & 66,7 & 100,0 & 66,7 & 63,3 & 0,587 & 0,322 \\
\hline Desvio Padrão & 43,7 & 40,3 & 43,5 & 42,3 & 39,1 & 27,6 & - & - \\
\hline
\end{tabular}

A intensidade da dor, nas cinco avaliações, pode ser observada na Tabela 3. 
Tabela 3 - Estatística descritiva e analítica da intensidade da dor, ao longo de 6 meses (São Paulo, 2000)

\begin{tabular}{|c|c|c|c|c|c|c|}
\hline \multirow[t]{2}{*}{ Avaliações } & \multicolumn{4}{|c|}{ Intensidade da dor } & \multicolumn{2}{|c|}{ Significância (P) } \\
\hline & Média & Mediana & $\begin{array}{l}\text { Desvio } \\
\text { padrão }\end{array}$ & Variação & ANOVA & Friedman \\
\hline $1^{a}$ & 8,3 & 9 & 2,0 & $3-10$ & - & - \\
\hline $2^{a}$ & 7,1 & 7 & 2,2 & $3-10$ & - & - \\
\hline $3^{a}$ & 6,9 & 7 & 2,4 & $1-10$ & $0,008^{*}$ & $0,003^{*}$ \\
\hline $4^{a}$ & 6,5 & 7 & 2,6 & $0-10$ & - & - \\
\hline $5^{a}$ & 6,6 & 7,3 & 3,0 & $0-10$ & - & - \\
\hline
\end{tabular}

${ }^{*} \mathrm{p}<0,05$ - estatísticamente significativo

A variável cultural locus de controle da saúde não apresentou variação estatisticamente significativa ao longo do tempo, isto é, o estilo de locus de controle da saúde permaneceu o mesmo, ao longo de 6 meses (Tabela 4). Os escores médios da subescala "internalidade" variaram entre 20,4 a 21,1; os escores médios da subescala "outros poderosos" variaram entre 21,3 a 22,6 e da subescala "acaso" entre 18,2 e 19,2. Os índices de "internalidade total" foram baixos, variaram entre 18,7 e 19,5 (a variação máxima no grupo foi 32). Tais dados podem ser observados na Tabela 4.

Tabela 4 - Estatística descritiva e analítica dos escores obtidos pela Escala de Locus de Controle da Saúde (LCS), nos 3 momentos de avaliação, ao longo de seis meses (São Paulo, 2000)

\begin{tabular}{l|c|c|c|c|c}
\hline Escore de LCS & \multicolumn{3}{|c|}{ Avaliação } & \multicolumn{2}{c}{ Significância (P) } \\
\hline & $1^{\mathrm{a}}$ & $3^{\mathrm{a}}$ & $5^{\mathrm{a}}$ & ANOVA & Friedman \\
\hline Internalidade & & & & & \\
$\quad$ Média & 20,4 & 20,4 & 21,1 & & \\
Mediana & 21,0 & 22,0 & 22,0 & 0,631 & 1,000 \\
Desvio padrão & 5,4 & 6,1 & 5,0 & & \\
Variação & $11,0-30,0$ & $8,0-30,0$ & $10,0-30,0$ & & \\
\hline Outros poderosos & & & & & \\
Média & 21,8 & 22,6 & 21,3 & & \multirow{2}{*}{0,186} \\
Mediana & 22,5 & 22,0 & 22,0 & 0,083 & \\
Desvio padrão & 5,8 & 5,2 & 5,6 & & \\
Variação & $11,0-30,0$ & $14,0-30,0$ & $10,0-30,0$ & & \\
\hline Acaso & & & & & \\
Média & 18,8 & 19,2 & 18,2 & & \multirow{2}{*}{0,871} \\
Mediana & 18,5 & 21,0 & 17,5 & 0,606 & \\
Desvio padrão & 7,9 & 8,4 & 8,1 & & \\
Variação & $7,0-30,0$ & $6,0-30,0$ & $6,0-30,0$ & & \\
\hline Internalidade total & & & & & \\
Média & 18,9 & 18,7 & 19,5 & & \\
Mediana & 19,0 & 20,0 & 19,3 & 0,632 & 0,825 \\
Desvio padrão & 6,4 & 7,6 & 5,8 & & \\
Variação & $9,5-32,0$ & $4,0-30,0$ & $6,0-30,0$ & & \\
\hline
\end{tabular}

Na Tabela 5 pode-se obsevar a as relações entre adesão e as escalas do Locus de Controle da Saúde. Segundo os Testes de correlação de Pearson e de Spearman, observou-se, na terceira avaliação, correlação negativa estatisticamente significativa entre a adesão e a subescala "internalidade" e o índice de "internalidade total". Isso significou que quanto maior a crença do doente de que o controle da sua saúde depende de si próprio, menor foi a adesão. 
Geana Paula Kurita Cibele A. de Mattos Pimenta

Tabela 5 - Coeficientes de correlação obtidos na análise da relação entre o escore de Locus de Controle da Saúde e a média do Índice de Acerto de Ingestão medicamentosa (IAIM) ao longo de seis meses (São Paulo, 2000)

\begin{tabular}{|c|c|c|c|c|}
\hline Avaliações & $\begin{array}{l}\text { Coeficiente de } \\
\text { correlação } \\
\text { Pearson (r) }\end{array}$ & $\begin{array}{c}\text { Significância } \\
(P)\end{array}$ & $\begin{array}{l}\text { Coeficiente de } \\
\text { correlação } \\
\text { Spearman }(r)\end{array}$ & $\begin{array}{c}\text { Significância } \\
(P)\end{array}$ \\
\hline $\begin{array}{l}\text { Internalidade } \\
1^{\mathrm{a}} \text { avaliação } \\
2^{\mathrm{a}} \text { avaliação } \\
3^{\mathrm{a}} \text { avaliação }\end{array}$ & $\begin{array}{l}-0,210 \\
-0,425 \\
-0,269\end{array}$ & $\begin{array}{l}0,266 \\
0,022^{*} \\
0,151\end{array}$ & $\begin{array}{l}-0,170 \\
-0,413 \\
-0,236\end{array}$ & $\begin{array}{c}0,368 \\
0,026^{*} \\
0,209\end{array}$ \\
\hline $\begin{array}{l}\text { Outros } \\
\text { poderosos } \\
\qquad 1^{\mathrm{a}} \text { avaliação } \\
2^{\mathrm{a}} \text { avaliação } \\
3^{\mathrm{a}} \text { avaliação }\end{array}$ & $\begin{array}{l}0,063 \\
-0,225 \\
-0,230\end{array}$ & $\begin{array}{l}0,741 \\
0,241 \\
0,222\end{array}$ & $\begin{array}{l}0,119 \\
-0,205 \\
-0,190\end{array}$ & $\begin{array}{l}0,533 \\
0,286 \\
0,314\end{array}$ \\
\hline $\begin{array}{l}\text { Acaso } \\
\qquad 1^{\mathrm{a}} \text { avaliação } \\
2^{\mathrm{a}} \text { avaliação } \\
3^{\mathrm{a}} \text { avaliação }\end{array}$ & $\begin{array}{l}-0,101 \\
-0,196 \\
-0,053\end{array}$ & $\begin{array}{l}0,597 \\
0,308 \\
0,783\end{array}$ & $\begin{array}{l}-0,126 \\
-0,211 \\
-0,030\end{array}$ & $\begin{array}{l}0,507 \\
0,271 \\
0,876\end{array}$ \\
\hline $\begin{array}{c}\text { Internalidade total } \\
1^{\mathrm{a}} \text { avaliação } \\
2^{\mathrm{a}} \text { avaliação } \\
3^{\mathrm{a}} \text { avaliação }\end{array}$ & $\begin{array}{l}-0,267 \\
-0,373 \\
-0,158\end{array}$ & $\begin{array}{c}0,153 \\
0,046^{*} \\
0,405\end{array}$ & $\begin{array}{l}-0,283 \\
-0,376 \\
-0,122\end{array}$ & $\begin{array}{c}0,129 \\
0,044^{*} \\
0,521\end{array}$ \\
\hline
\end{tabular}

\section{DISCUSSÃO}

Observou-se que a adesão ao tratamento foi insuficiente e não variou ao longo de seis meses. Supunha-se que a adesão seria mais elevada no início e diminuiria com o tempo, o que não ocorreu. Os índices de não adesão e de acerto de ingestão medicamentosa observados assemelharam-se aos encontrados em outros trabalhos ${ }^{(8-10)}$, que também descrevem valores entre $30 \%$ e $70 \%$ de não adesão ou adesão parcial. Tais dados são preocupantes e indicam a necessidade de intervenções que melhorem a adesão.

A intensidade da dor diminuiu ao longo do tratamento e essa diferença foi estatisticamente significativa $(8,3$ na primeira avaliação e 6,5 foi a menor média foi). Apesar de estatisticamente diferente, é possível que a expressão clínica dessa diferença seja modesta, isto é, a dor pouco aliviou, e isto contribuíu para a manutenção dos altos índices de não adesão e adesão parcial.

Para se mensurar a adesão ao tratamento não há procedimento padrão. Há na literatura a descrição de quatro possibilidades: o autorelato, a observação do comportamento, testes laboratoriais com fluídos corpóreos para verificar o nível plasmático dos medicamentos ingeridos e a observação de resultados clínicos ${ }^{(11)}$. Entretanto, nenhum desses métodos está isento de erro. O autorelato, embora freqüentemente utilizado, nem sempre é acurado. O doente pode subestimar ou superestimar sua adesão, por fatores sociais, emocionais ou mesmo cognitivos (alteração na atenção, memória, etc.). A observação de comportamento mais comumente utilizada para avaliar a adesão é a contagem do medicamento, no entanto, não há garantia de que as pílulas realmente tenham sido ingeridas. Em relação a monitorização de exames laboratoriais, a ingestão a poucas horas do exame ou a interrupção recente, dependendo da meia vida da droga, resulta em falsos resultados, além de ser um serviço caro e nem sempre disponível. E a observação de resultados clínicos nem sempre apresenta uma associação linear entre resultados e adesão.

Outra dificuldade para se avaliar a adesão ao tratamento da dor crônica é a falta de consenso sobre qual critério de sucesso deve ser utilizado para mensurá-la, o que limita metodologicamente a comparação dos poucos estudos existentes sobre o tema.

A não adesão pode resultar em progressão da doença, piora da qualidade de vida, ônus econômico ao indivíduo, ao serviço de saúde e à sociedade e fator de confusão nas pesquisas que testam intervenções. $\mathrm{O}$ comportamento de adesão é multifatorial, 
complexo e difícil de ser avaliado. Relacionase às características da doença, do tratamento, dos profissionais envolvidos e do perfil psicocultural dos doentes.

Foram feitas três avaliações de locus de controle da saúde ao longo de seis meses, e não se observou variação estatisticamente significativa nesse período.

Há dúvidas de que o locus de controle seja um atributo com características de traço ou de estado. Tamayo(7) cita que há uma tendência em simplificar o locus de controle e considerá-lo como um traço permanente do indivíduo, talvez porque a origem da mensuração tenha sido feita por meio de escalas unifatoriais. No entanto, é um construto muito mais dinâmico, com dimensões cognitiva, perceptiva, motivacional e existencial e com variações na orientação, dependendo da situação $0^{(7)}$. Assim sendo, há uma possibilidade de mudança na orientação do locus ao longo do tempo, o que justifica as avaliações seqüenciais.

Seguindo-se a orientação de que quanto maior o escore, maior é a crença em uma dada subescala, a maior média (entre 21,3 e 22,6) pertenceu à subescala "outros poderosos", revelando que os doentes possuem crença maior em fontes externas de poder, ou seja, que pessoas poderosas (médico, autoridades, etc.) controlam seu estado de saúde. A crença no "Acaso" (entre 18,2 e 19,2) foi menor do que na "Internalidade" $(20,4$ e 21,1) e "Outros Poderosos".

$\mathrm{Na}$ presente pesquisa observou-se correlação negativa entre a subescala "internalidade" $(\mathrm{P}=0,022$ e 0,026$)$, o índice de "internalidade total" $(\mathrm{P}=0,046$ e 0,044$) \mathrm{e}$ o Índice de Acerto de Ingestão de Medicamentos. Isto é, quanto maior a crença do doente de que o controle de sua saúde dependia de si próprio, menor foi a adesão e esse dado causou certa surpresa.

Tinha-se como hipótese de que maior "internalidade" estaria relacionada a uma maior adesão, o que não foi observado. Para a análise desses resultados cabe considerar que os doentes do presente estudo tinham dor em média há 57 meses e obtiveram alívio da dor modesto (melhor média de intensidade de dor foi 6,5, em escala de 0 a 10 - Tabela 3 ) e que o tratamento foi prolongado (6 meses). É possível que o doente com orientação mais interna tenha avaliado a situação (pequena melhora e longo tempo de tratamento) e tomou a decisão de interromper a terapia, o que representou menor adesão. Talvez doentes com orientação mais externa sejam mais obedientes, independentes da avaliação particular da situação. Há controvérsias sobre qual a orientação desejável do locus de controle nas questões de saúde ${ }^{(5)}$.

Há poucos estudos que analisam o locus de controle da saúde em doentes com dor crônica.

Em estudo comparativo com 89 doentes com lombalgia crônica, realizado por Friedrich, Gittler, Halberstadt, Cermak e Heiller na Aústria $^{(12)}$, observou-se que, tanto no grupo controle (exercícios físicos) quanto no grupo experimental (exercícios físicos e intervenções motivacionais), houve maior escore para o estilo de locus interno na primeira avaliação (três semanas e meia de seguimento) e redução até a última avaliação (12 meses). As médias iniciais foram 13,2 e 13,4 para o grupo experimental e 12,5 e 12,6 para o grupo controle nas primeiras avaliações, e não houve diferença significativa entre os grupos. Aos doze meses de seguimento, as médias reduziram-se para 11,8 para o grupo experimental e 11,2 para o controle. O estudo aponta também que adesão aos exercícios ocorreu em indivíduos com maiores escores de locus de controle interno. Tais resultados diferem dos observados no presente estudo, sendo observado maior crença em "outros poderosos" e que maior "internalidade" representou menor adesão ao tratamento. Não está descrito no estudo citado ${ }^{(12)}$ o método de avaliação de adesão e nem o alívio da dor que os doentes obtiveram. É possível que diferenças nesses aspectos tenham contribuído para as divergências observadas.

A relação entre locus de controle, estratégias de coping e distress (tristeza) psíquico em doentes com dor crônica foi estudada e os resultados mostraram que doentes com orientação externa, voltada para a "sorte e o acaso", tenderam a confiar em estratégias de coping mal adaptativas e suas habilidades para controlar a dor foram pobres ${ }^{(13)}$. Os doentes preferiram utilizar estratégias como desviar a atenção e rezar para lidar com a dor. Também exibiram grande distress psíquico. Esses autores consideram
Adesão ao tratamento da dor crônica e o locus de contrôle da saúde 
Geana Paula Kurita Cibele A. de Mattos Pimenta que os doentes com orientação para a "sorte", freqüentemente relatam depressão, ansiedade e sintomas obsessivo-compulsivos. Consequentemente, relatam a desesperança que sentem para lidar efetivamente com a dor.

Estudos relatam que doentes com orientação externa "acaso" acreditaram que fatores como o destino e o tempo eram responsáveis pela dor. Doentes com estilo de locus voltado para "outros poderosos" acreditaram que a visita ao médico e a rigorosa adesão ao tratamento prescrito eram os melhores meios para o controle da dor. Observaram-se algumas diferenças significativas entre o sexo, a idade, o estilo de locus de controle e a utilização de estratégias de coping. Homens mais jovens tiveram estilo de locus de controle interno, enquanto os mais velhos tiveram orientação externa. Mulheres com locus de controle interno fizeram maior uso das estratégias de coping em relação as mulheres com outra orientação de locus $^{(14)}$.

Em estudo com 100 doentes com dor crônica com o intuito de avaliar fatores preditivos de descontinuação do tratamento, os doentes assistiram a um videotape de 15 minutos sobre diferenciação entre dor crônica e aguda, descrição do tratamento conservador para dor crônica e do tratamento que a clínica oferecia. Após o vídeo, responderam a um questionário que mensurava a informação captada e a sua aceitação ou rejeição. Não houve diferença quanto ao estilo de locus de controle da saúde e a concordância com as informações apresentadas no vídeo, isto é, as crenças dos doentes não sobressaíram como fator preditivo de não adesão ${ }^{(15)}$.

Há outros estudos sobre a crença de controle da saúde em doentes com outros agravos, descritos a seguir.

Arakelian ${ }^{(16)}$ expôs suas idéias sobre locus de controle e adesão ao tratamento da obesidade. Acredita que pessoas com locus de controle externo tenham maior propensão para adiar cirurgias corretivas e quando participam de programas de controle de peso, são as de menor sucesso. Cita também diferenças em indivíduos com locus de controle com orientação externa e interna em relação ao tratamento. Apesar de a "internalidade" ser a perspectiva mais desejável, de modo genérico, pessoas com locus de controle interno negam-se a aceitar o tratamento. Em contraste, indivíduos com locus de controle externo têm maior índice de aprovação e sentem-se menos responsáveis pelos resultados do tratamento, especialmente os desfavoráveis, e preferem situações que lhes facilitem racionalizar insucessos e resultados que dependem muito mais da sorte do que das habilidades pessoais.

O locus de controle também foi objeto de uma revisão de literatura para se avaliar as diferenças culturais entre grupos de latinos e anglo-saxãos, e as implicações no cuidado primário e prática de saúde pública. A prática cultural americana e o conceito de prevenção de doenças e promoção da saúde são compatíveis com a dimensão interna, através da qual o indivíduo acredita controlar os eventos de sua vida, incluindo a habilidade para prevenir certas condições de saúde. Pessoas com essa orientação tendem a não ver esses eventos com fatalismo. Já as crenças tradicionais latinas são congruentes com a dimensão externa que evoca o fatalismo e crenças na sorte, no "acaso" e em Deus como determinantes de saúde e doença. Nessa visão, as pessoas com locus de controle externo são vistas como em risco para desenvolverem doenças devido à falta de cuidados apropriados e problemas de adesão às intervenções médicas e de saúde pública $^{(17)}$.

Indivíduos com estado de saúde precário podem viver situações de impotência causadas por diversos fatores, que incluem mudanças relacionadas à doença até a interação com a equipe de saúde. O locus de controle influencia o comportamento do doente diante do problema de saúde, direcionando a percepção do indivíduo para fatores dependentes de si ou de forças externas. Conhecer a orientação do locus de controle do doente é importante para que se antecipem as mudanças que o ele necessitará fazer tendo em vista o melhor manejo do tratamento ${ }^{(18)}$.

É importante ter-se em mente a falta de sustentação empírica do locus de controle da saúde. Há controvérsias sobre a orientação desejável e a falta de dados sobre diversos fatores como o comportamento prévio, o suporte social, a motivação, a percepção dos custos, os benefícios das ações e as atitudes em relação aos profissionais de saúde, entre outros, que compõem o estilo de locus de controle ${ }^{(5)}$. 


\section{CONCLUSÃO}

O estudo permitiu concluir que os índices de adesão parcial e não adesão ao tratamento medicamentoso foram altos, que o índice de acerto de ingestão medicamentosa foi baixo, cujos valores não oscilaram ao longo de seis meses. Os valores de "internalidade total" do locus de controle e o de "outros poderosos" demonstraram doentes com tendência a crer que o cuidado à saúde depende mais de outros do que de si próprio. Constatou-se a influência da dimensão "internalidade" e do índice de "internalidade total" (LCS) na adesão ao tratamento da dor crônica, isto é, os doentes com maior "internalidade" aderiram menos ao tratamento. Tais resultados demonstram a importância das crenças no manejo do tratamento da dor crônica e que intervenções específicas, visando incrementar a adesão, devem ser testadas.

\section{REFERÊNCIAS}

(1) Kolton KA, Piccolo P. Patient compliance: a challenge in practice. Nurse Pract 1988; 13(12):37-50.

(2) Dela Coleta MF. Escala multidimensional de locus de controle de Levenson. Arq Bras Psicol 1987; 39(2):79-97.

(3) Turk DC, Meichenbaum D, Genest M. Pain and behavioral medicine: a cognitive behavioral perspective. New York: Guilford; 1983.

(4) Huskisson EC. Measurament of pain. Lancet 1974; 2(7889):1127-31.

(5) Wallston KA, Wallston BS, DeVellis R. Development of the Multidimensional Health Locus of Control (MHLC) Scales. Health Educ Monogr 1978; 6(2):160-70.

(6) Levenson, H. Multidimensional locus of control in psychiatric patients. J Consult Clin Psychol 1973; 41(3):397-404.

(7) Tamayo A. Validação fatorial da escala Levenson de locus de controle. Psicol: teor e pesqui 1989; 5(1):111-22.

(8) Berndt S, Maier C, Schütz HW. Polymedication and medication compliance in patients with chronic non-malignant pain. Pain 1993; 52(3):331-9.

(9) Packar RC, O' Connell P. Medication compliance among headache patients. Headache 1986; 26:416-9.

(10) Pimenta CAM. Aspectos culturais, afetivos e terapêuticos relacionados à dor no câncer. [tese] São Paulo (SP): Escola de Enfermagem da USP; 1995.
(11) Turk DC, Rudy TE. Neglected topics in the treatment of chronic pain patients: relapse, noncompliance, and adherence enhancement. Pain 1991; 44(1):5-28.

(12) Friedrich M, Gittler G, Halberstadt Y, Cermak T, Heiller I. Combined exercise and motivation program: effect on the compliance and level of disability of patients with chronic low back pain: a randomized controlled trial. Arch Phys Med Rehabil 1998; 79(5):475-87.

(13) Crisson JE, Keefe FJ. The relationship of locus of control to pain coping strategies and psychological distress in chronic pain patients. Pain 1988; 35(2):147-54.

(14) Buckelew SP, Shutty MS, Heweett J, Landon T, Morrow K, Frank RG. Health locus of control, gender differences and adjustment to persistent pain. Pain 1990; 42(3):287-94.

(15) Shutty MS, DeGood DE, Tuttle DH. Chronic pain patients' beliefs about their pain and treatment outcomes. Arch Phys Med Rehabil 1990; 71(2):128-32.

(16) Arakelian M. An assessment and nursing application of the concept of locus of control. Adv Nurs Sci 1980; 3(1):25-42.

(17) Talavera GA, Elder JP, Velasquez RJ. Latino health beliefs and locus of control: implications for primary care and public health practitioners. Am J Prev Med 1997; 13(6):408-10.

(18) Schroeder PS, Miller JF. Qualitative study of locus of control in patients with peripheral vascular disease. In: Miller JF. Coping with chronic illness: overcoming powerlessness. Philadelphia: FA Davis; 1983. p. 149-61.
Adesão ao tratamento da dor crônica e o locus de contrôle da saúde 